\title{
Crescimento e estruturas do sistema digestório de larvas de pacu alimentadas com dieta microencapsulada produzida experimentalmente
}

\author{
Olívia Cristina Camilo Menossi ${ }^{1}$, Rodrigo Takata ${ }^{1}$, María Isabel Sánchez-Amaya ${ }^{2}$, Thiago \\ Mendes de Freitas ${ }^{1}$, Manuel Yúfera ${ }^{2}$, Maria Célia Portella ${ }^{1}$
}

${ }^{1}$ Centro de Aquicultura da Unesp, Brasil.

2 Instituto de Ciencias Marinas de Andalucia (ICMAN-CSIC), Espanha.

RESUMO - Foram comparados os efeitos de diferentes dietas e da transição alimentar sobre o desempenho zootécnico e a morfologia do trato digestório de larvas de pacu alimentadas com duas dietas comerciais e uma dieta experimental microencapsulada produzida por gelificação interna. Larvas com quatro dias de vida receberam os seguintes protocolos alimentares: somente náuplios de artêmia em quantidades crescentes (controle positivo); larvas mantidas em jejum (controle negativo); três tipos de alimento formulado durante todo o experimento (dieta experimental microencapsulada, dieta comercial NRD1.2/2.0, Inve, USA, e dieta Poli-Peixe 450F, PoliNutri, Brasil); e três protocolos de transição alimentar com náuplios de artêmia durante os seis primeiros dias, seis dias de coalimentação, e somente as respectivas dietas formuladas após esse período. O experimento foi conduzido por 23 dias em delineamento inteiramente casualizado, com avaliações periódicas do crescimento e do sistema digestório. A taxa de sobrevivência foi determinada ao final do experimento. Os melhores desempenhos de crescimento e sobrevivência, assim como mais acelerada organogênese do sistema digestório, foram verificados entre os animais que receberam alimento vivo. As larvas que passaram pela transição alimentar apresentaram médias intermediárias de crescimento e sobrevivência e não diferiram entre si. Entre os grupos que receberam apenas dieta inerte, somente naquele alimentado com a dieta nacional houve sobrevivência até o final do experimento. Nenhuma das dietas formuladas é adequada para uso como alimento exclusivo para larvas de pacu. Na fase de transição alimentar, a dieta experimental microencapsulada proporciona resultados de sobrevivência e crescimento semelhantes aos obtidos com dietas comerciais.

Palavras-chave: histologia, larvicultura, microencapsulação, organogênese, Piaractus mesopotamicus, transição alimentar

\section{Growth and structure of the digestive system of pacu larvae fed microencapsulated diet produced experimentally}

\begin{abstract}
The effects of different diets and dietary transition on the performance and morphology of the digestive tract of pacu larvae fed two commercial formulated diets and an experimental microencapsulated diet produced by internal gelation were evaluated. Four-day old pacu larvae received the following treatments: only artemia nauplii in increasing amount during the experiment (positive control); larvae maintained at fasting (negative control); three types of formulated diets throughout the experiment (experimental microencapsulated diet, commercial diet NRD1.2/2.0, Inve, USA, and diet PoliPeixe 450F, PoliNutri, Brazil); and three weaning protocols in which artemia nauplii were given from the $1^{\text {st }}$ to $6^{\text {th }}$ days, six days of co-feeding (artemia nauplii + the respective formulated diet), and only respective diets formulated after this period. The experiment was conducted for 23 days in a completely randomized design, with periodical evaluations of growth and digestive system. Survival rate was determined at the end of the experiment.The best performances of growth and survival, as well as the organogenesis of the digestive tract, were verified for the animals which received live feed. The larvae at weaning treatments induced intermediate growth and survival, which did not differ statistically between them. Considering the treatments that received only formulated diets as first feed, the PoliNutri diet was the unique that showed alive larvae at the end of experiment. None of the formulated diets are adequate as initial exogenous food for pacu larvae. During the weaning, the microencapsulated experimental diet provides growth and survival performances similar to those obtained with the commercial diets.
\end{abstract}

Key Words: histology, larviculture, microencapsulation, organogenesis, Piaractus mesopotamicus, weaning

\section{Introdução}

Inúmeros protocolos de transição alimentar têm sido testados para a larvicultura intensiva de peixes visando reduzir o período de fornecimento do alimento vivo (Hart \& Purser, 1996; Jomori, 2005; Jomori et al. 2008; Curnow et al. 2006; Kestemont \& Stalmans 1992), porém, devido a diversos fatores, como imaturidade do sistema digestório das larvas, 
formulação e processamento das dietas, o uso de microdietas como primeira fonte de alimento não tem proporcionado resultados satisfatórios (Portella \& Dabrowski, 2008).

Além de nutricionalmente balanceado, o alimento inerte também deve ser atrativo, palatável e apresentar densidade adequada e estabilidade na água. Todavia, devido ao tamanho reduzido das partículas, muitos nutrientes são lixiviados em contato com a água. Visando solucionar esses problemas, novas tecnologias de produção de microdietas são alvo de estudos objetivando obter partículas mais estáveis. Entre os métodos aplicados na produção de dietas adequadas e nutricionalmente completas para larvas de peixes, destaca-se o processo de microencapsulação. A microencapsulação pode ser obtida por vários processos, como a gelificação iônica, coacervação simples ou complexa, spray-drying, entre outros (Yúfera et al., 2009).

Há alguns anos, Yúfera et al. (1999) desenvolveram uma dieta microencapsulada para larvas de peixes produzida pelo método de polimerização interfacial. Entretanto, devido ao alto custo da produção e ao uso de componentes tóxicos para seu preparo, os métodos foram aperfeiçoados a fim de se produzir uma dieta mais viável e ambientalmente amigável. O uso da técnica de gelificação iônica interna proposto por Yúfera et al. (2005) resulta em partículas mais adequadas para uso na aquicultura, uma vez que o custo da produção da dieta é mais baixo e não exige solventes tóxicos.

Considerando os resultados promissores obtidos por Yúfera et al. (2005) com o uso dessa dieta para larvas das espécies marinhas Spaurus aurata e Solea senegalensis, objetivou-se com este estudo avaliar essa dieta para uma espécie de peixe neotropical, o pacu (Piaractus mesopotamicus), cujas larvas também possuem características altriciais, assim como a maioria das larvas de peixes marinhos. $\mathrm{Na}$ avaliação, foram comparados $\mathrm{o}$ desempenho, as alterações morfológicas do trato digestório e os efeitos da transição alimentar das larvas alimentadas com duas dietas comerciais e uma dieta experimental microencapsulada produzida por gelificação interna.

\section{Material e Métodos}

As larvas de pacu (Piaractus mesopotamicus) foram obtidas por reprodução induzida de um único casal. Aos 4 dias de vida $(5,74 \pm 0,17 \mathrm{~mm}$ de comprimento total e $0,62 \pm 0,13 \mathrm{mg}$ de peso úmido total), as larvas foram contadas individualmente e estocadas (12 larvas/L) em 30 tanques de polietileno com $50 \mathrm{~L}$ de água proveniente de poço artesiano, com fluxo contínuo e aeração artificial constante. Durante o período experimental (23 dias), as médias de oxigênio dissolvido mantiveram-se em $6,3 \pm 0,2 \mathrm{mg} / \mathrm{L}$ e $78 \%$ de saturação. As temperaturas médias no período da manhã e tarde foram de $28,2 \pm 0,6^{\circ} \mathrm{C}$ e $29 \pm 0,7^{\circ} \mathrm{C}$, respectivamente.

Para análise estatística, considerou-se um delineamento inteiramente casualizado, constituído de oito tratamentos, cada um com quatro réplicas: controle positivo = náuplios de artêmia em quantidade crescente por todo período experimental; controle negativo $=$ larvas mantidas em jejum por todo período experimental; dieta comercial NRD1.2/2.0 (Inve, USA) durante todo o período experimental; dieta comercial Poli-Peixe diet 450F (PoliNutri, Brasil) durante todo o período experimental; dieta experimental microencapsulada produzida segundo Yúfera et al. (2005) durante todo o experimento; três tratamentos com transição alimentar utilizando-se náuplios de artêmia durante os seis primeiros dias experimentais, seis dias de coalimentação e somente as respectivas dietas formuladas após esse período. Segundo informações presentes nos rótulos, a dieta PoliPeixe 450 F, PoliNutri, continha 45\% de proteína bruta (PB), enquanto a dieta importada NRD1.2/2.0-Inve, 57\%. A dieta microencapsulada, com aproximadamente $51 \%$ de PB, foi produzida no Instituto de Ciencias Marinas de Andalucía (CSIC), Espanha, e continha, em g/kg: caseína, 260; farinha de peixe, 155; farinha de lula, 145; hidrolisado de peixe, 125; emulsão de óleo, 135; lecitina de soja, 30; levedura, 50; vitamina C, 30; vitamina E, 10; e alginato de sódio, 60 .

Nos grupos alimentados com as dietas comerciais e a dieta microencapsulada após transição alimentar, os náuplios de artêmia foram fornecidos em quantidades crescentes nos primeiros seis dias. Após esse período, houve supressão gradual do alimento vivo com introdução simultânea das dietas formuladas até a retirada total dos náuplios (Figura 1A). As larvas do controle positivo receberam náuplios de artêmia em quantidade crescente durante todo período experimental (Figura 1B). As dietas formuladas foram fornecidas em excesso, formando um filme que cobria toda a superfície do tanque (100 a 200 mg). As quantidades diárias de ração e de alimento vivo foram fracionadas em cinco refeições, às $8,11,14,17$ e $20 \mathrm{~h}$. Todas as dietas apresentavam partículas de tamanho $<200 \mu \mathrm{m}$. Após a alimentação das $8 \mathrm{~h}$, era realizado o sifonamento dos tanques para retirada de eventuais larvas mortas e restos alimentares.

Amostras de larva de cada réplica foram coletadas ao $6^{\circ}, 12^{\circ}, 18^{\circ}$ e $23^{\circ}$ dias experimentais, para avaliação do crescimento $(n=30)$ e análises histológicas $(n=5)$. As amostras foram fixadas em formol 10\% tamponado e preservadas em álcool a 70\%. Com os dados de peso, foi calculada a taxa de crescimento específico utilizando-se a seguinte expressão:

$\mathrm{TCE}=($ Ln peso final - Ln peso inicial $) \times 100 /$ intervalo entre biometrias (dias). 


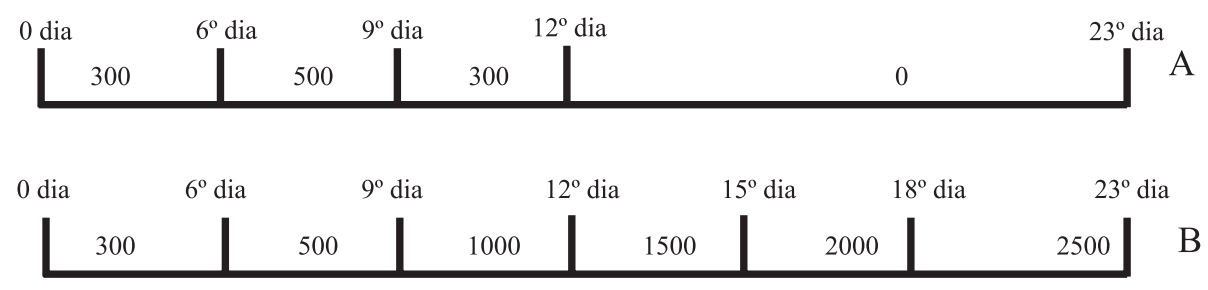

$\mathrm{A}$ = que passaram pela transição alimentar; $\mathrm{B}=$ que receberam náuplios de artêmia em quantidades crescentes por todo período experimental.

Figura 1 - Representação esquemática das quantidades diárias de alimento vivo (náuplios de artêmia por larva) oferecidas às larvas de pacu durante os dias experimentais.

A determinação da taxa de sobrevivência foi realizada no final do experimento, quando se realizou a contagem individual dos animais sobreviventes. Os resultados de sobrevivência e desempenho zootécnico (peso e comprimento) foram submetidos à análise de variância e ao teste Tukey-Kramer, a 5\% de probabilidade, e analisados pelo programa SAS System, versão 8.0. Os resultados percentuais das taxas de sobrevivência e crescimento específico passaram pela transformação $\mathrm{y}=\operatorname{arcsen} \sqrt{x / 100}$, de modo que $\mathrm{x}$ era o valor expresso em porcentagem, antes da aplicação da ANOVA. Todas as variâncias biométricas foram submetidas a testes para verificação da normalidade (Cramer Von Mises) e homocedasticidade das variâncias (Levene).

Para os procedimentos histológicos, as larvas foram incluídas em Historesina ${ }^{\circledR}$ (Leica, Germany). Foram obtidos cortes de $3 \mu \mathrm{m}$ de espessura com a utilização de micrótomo automático LEICA, modelo RM 2155, utilizando-se navalhas de vidro, que foram corados pelos métodos hematoxilinaeosina, ácido periódico de Schiff, eosina-orange G-floxina e azul de metileno.

\section{Resultados e Discussão}

Os resultados de sobrevivência foram influenciados pelos alimentos e pelos esquemas alimentares (Figura 2). O fornecimento exclusivo de alimento vivo proporcionou a maior média $(\mathrm{P}<0,05)$ ao final do experimento $(69,7 \pm 5,2 \%)$. Os grupos que passaram por transição alimentar apresentaram taxas de sobrevivência intermediárias e não diferiram entre si $(\mathrm{P}>0,05)$. A menor média $(\mathrm{P}<0,05)$ foi verificada nas larvas que receberam ração comercial nacional $(9,9 \pm 5,5 \%)$, porém, entre os grupos que receberam a dieta inerte desde o início do experimento, esse foi o único que proporcionou larvas vivas no final do estudo.

A mortalidade massiva das larvas que recebiam a dieta inerte importada e a microencapsulada sem transição alimentar com náuplios de artêmia ocorreu no $18^{\circ}$ dia experimental, próximo ao do grupo controle negativo de

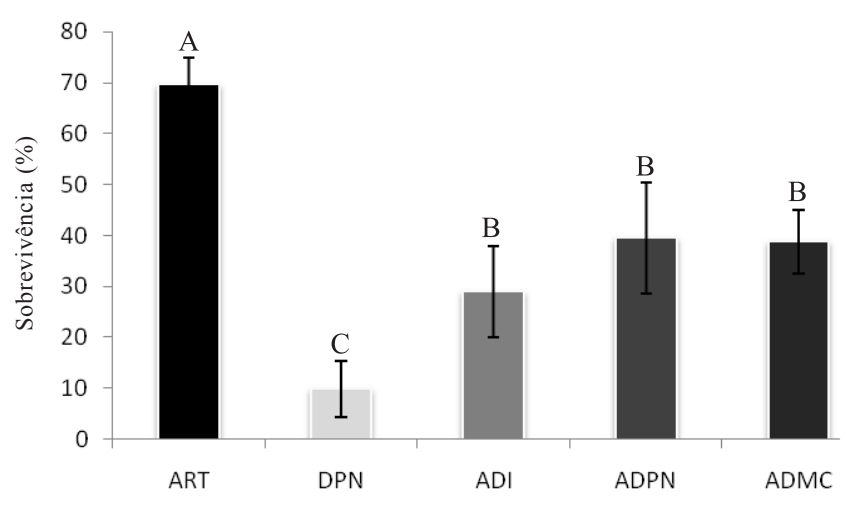

$\mathrm{ART}=$ náuplios de artêmia por todo período experimental; DPN $=$ dieta comercial nacional por todo o período experimental; $\mathrm{ADI}, \mathrm{ADPN}$ e $\mathrm{ADMC}=$ náuplios de artêmia durante os seis primeiros dias experimentais, seis dias de coalimentação e, após esse período, somente as dietas comercial importada, comercial nacional e experimental microencapsulada, respectivamente.

Figura 2 - Valores médios (\%) da taxa de sobrevivência de larvas de pacu submetidas a diferentes sistemas de alimentação.

larvas em jejum, que morreram no 15 o dia. Assim, as poucas larvas restantes alimentadas com a dieta comercial importada e a dieta experimental microencapsulada foram coletadas para avaliação de crescimento e desenvolvimento do tubo digestivo e esses tratamentos foram encerrados. Vários fatores podem ter contribuído para esse resultado, como baixa ingestão da dieta, composição nutricional inadequada para assegurar bom desenvolvimento larval e imaturidade do sistema digestório para digerir e assimilar os nutrientes presentes na dieta. Pode-se supor que a dieta microencapsulada e a importada fornecida sem a transição com náuplios de artêmia contribuíram minimamente com algum aporte nutricional que proporcionou maior tempo de vida às larvas, em comparação às mantidas em jejum. Todavia, as dietas, evidentemente, foram inadequadas para o desenvolvimento das larvas, que morreram por inanição. Resultados similares foram relatados por Tesser et al. (2005), que verificaram mortalidade total ao $16^{0}$ dia de vida em larvas de $P$. mesopotamicus alimentadas exclusivamente com dieta microencapsulada comercial, que coincidiu com a mortalidade das larvas em jejum, comprovando ineficiência 
da dieta como de primeiro alimento para larvas de pacu. Por outro lado, resultados de um estudo realizado por Jomori et al. (2008), também com larvas de pacu, sinalizaram, pela primeira vez, a possibilidade de uso exclusivo de uma dieta comercial formulada para larvas de pacu em substituição total ao alimento vivo. Como neste estudo, mesmo com prejuízo do crescimento e da sobrevivência, os autores relataram $11 \%$ de sobrevivência entre os animais que recebiam apenas a dieta comercial. Muitos estudos ressaltam a dificuldade de uso de dietas formuladas para larvas altriciais de espécies marinhas (Cahu \& Zambonino Infante, 2001; Kolkovski, 2001; Koven et al., 2001). Todavia, Fernández-Díaz \& Yúfera (1997) relataram sobrevivência de $11 \%$ de larvas de S. aurata ao final do período experimental (15 dias) apenas com uso de dieta microencapsulada como fonte de alimento, indicando que a substituição total, mesmo sendo possível, ainda é inviável em termos produtivos.

Os maiores valores médios de peso (Tabela 1) e comprimento (Tabela 2) foram observados entre as larvas que receberam alimento vivo por todo o período experimental, seguido pelas larvas que foram submetidas à transição gradual do alimento vivo para o formulado. Os grupos que receberam as dietas inertes desde o início do experimento apresentaram as menores médias desses parâmetros.

Até os seis dias, as médias de comprimento total, peso e taxa de crescimento específico das larvas que recebiam alimento vivo não diferiam entre si, pois as larvas estavam recebendo as mesmas quantidades de artêmia (Tabelas $1 \mathrm{e}$ 2; Figura 3). Todavia, foram significativamente maiores que os das larvas que recebiam somente as dietas inertes (Tabelas 1 e 2; Figura 3).

Com a supressão total do alimento vivo (a partir do $13^{\circ}$ dia), as larvas que passaram pela transição alimentar apresentaram menores médias de peso e comprimento total e diferiram estatisticamente daquelas que ainda recebiam apenas alimento vivo (Tabelas 1 e 2). A estagnação do crescimento em peso observada nas larvas em transição alimentar após a retirada total do alimento vivo já foi relatada para larvas de pacu: Tesser et al. (2006) submeteram larvas de pacu a transição alimentar abrupta após cinco dias de fornecimento de náuplios de artêmia e observaram que praticamente não houve aumento de peso e comprimento durante alguns dias, porém, após esse período, houve tendência de recuperação. Da mesma forma, Jomori et al. (2003) e Jomori (2005) detectaram estagnação do peso e do

Tabela 1 - Peso úmido (mg) de larvas de pacu submetidas a diferentes sistemas de alimentação

\begin{tabular}{|c|c|c|c|c|}
\hline \multirow[b]{2}{*}{ Tratamento } & \multicolumn{4}{|c|}{ Dias após o início do experimento } \\
\hline & 6 dias & 12 dias & 18 dias & 23 dias \\
\hline ART & $1,24 \pm 0,03 \mathrm{~A}$ & $5,02 \pm 0,15 \mathrm{~A}$ & $14,19 \pm 0,85 \mathrm{~A}$ & $51,82 \pm 2,53 \mathrm{~A}$ \\
\hline JJ & $0,49 \pm 0,08 \mathrm{~B}$ & $0,24 \pm 0,02 \mathrm{D}$ & - & - \\
\hline DI & $0,55 \pm 0,02 \mathrm{~B}$ & $0,34 \pm 0,05 \mathrm{D}$ & $0,50 \pm 0,05 \mathrm{D}$ & - \\
\hline DPN & $0,53 \pm 0,02 \mathrm{~B}$ & $0,46 \pm 0,08 \mathrm{D}$ & $0,62 \pm 0,16 \mathrm{D}$ & $0,56 \pm 0,04 \mathrm{C}$ \\
\hline DMC & $0,52 \pm 0,02 \mathrm{~B}$ & $0,35 \pm 0,04 \mathrm{D}$ & $0,70 \pm 0,15 \mathrm{D}$ & - \\
\hline ADI & $1,23 \pm 0,02 \mathrm{~A}$ & $3,13 \pm 0,18 \mathrm{~B}$ & $3,47 \pm 0,34 \mathrm{~B}$ & $6,30 \pm 1,37 \mathrm{~B}$ \\
\hline ADPN & $1,23 \pm 0,02 \mathrm{~A}$ & $2,76 \pm 0,12 \mathrm{C}$ & $2,48 \pm 0,29 \mathrm{C}$ & $5,20 \pm 0,84 \mathrm{~B}$ \\
\hline ADMC & $1,25 \pm 0,02 \mathrm{~A}$ & $3,33 \pm 0,16 \mathrm{~B}$ & $3,05 \pm 0,28 \mathrm{BC}$ & $5,48 \pm 0,70 \mathrm{~B}$ \\
\hline
\end{tabular}

Médias seguidas de mesma letra na vertical não diferem $(\mathrm{P}<0,05)$ entre si pelo teste Tukey.

$\mathrm{ART}=$ alimento vivo por todo período experimental; $\mathrm{JJ}=$ jejum; DI $=$ dieta comercial importada; DPN $=$ dieta comercial nacional; DMC $=$ dieta experimental microencapsulada; ADI, ADPN e ADMC = náuplios de artêmia durante os seis primeiros dias experimentais, seis dias de coalimentação, e somente as respectivas dietas formuladas após esse período.

Tabela 2 - Comprimento total $(\mathrm{mm})$ de larvas de pacu submetidas aos diferentes sistemas de alimentação

\begin{tabular}{|c|c|c|c|c|}
\hline \multirow[b]{2}{*}{ Tratamento } & \multicolumn{4}{|c|}{ Dias após o início do experimento } \\
\hline & 6 dias & 12 dias & 18 dias & 23 dias \\
\hline ART & $6,67 \pm 0,02 \mathrm{~A}$ & $9,30 \pm 0,14 \mathrm{~A}$ & $9,63 \pm 0,22 \mathrm{~A}$ & $15,73 \pm 0,29 \mathrm{~A}$ \\
\hline JJ & $5,74 \pm 0,01 \mathrm{~B}$ & $5,73 \pm 0,04 \mathrm{C}$ & - & - \\
\hline DI & $5,77 \pm 0,02 \mathrm{~B}$ & $5,84 \pm 0,03 \mathrm{C}$ & $5,79 \pm 0,14 \mathrm{C}$ & - \\
\hline DPN & $5,77 \pm 0,01 \mathrm{~B}$ & $5,77 \pm 0,07 \mathrm{C}$ & $5,89 \pm 0,16 \mathrm{C}$ & $6,84 \pm 0,28 \mathrm{C}$ \\
\hline DMC & $5,73 \pm 0,03 \mathrm{~B}$ & $5,72 \pm 0,07 \mathrm{C}$ & $5,73 \pm 0,05 \mathrm{C}$ & - \\
\hline ADI & $6,96 \pm 0,04 \mathrm{~A}$ & $8,81 \pm 0,15 \mathrm{~B}$ & $9,25 \pm 0,33 \mathrm{~B}$ & $10,43 \pm 0,26 \mathrm{~B}$ \\
\hline ADPN & $6,90 \pm 0,03 \mathrm{~A}$ & $8,86 \pm 0,13 \mathrm{~B}$ & $9,24 \pm 0,16 \mathrm{~B}$ & $10,11 \pm 0,31 \mathrm{~B}$ \\
\hline ADMC & $6,92 \pm 0,02 \mathrm{~A}$ & $9,00 \pm 0,08 \mathrm{~B}$ & $9,49 \pm 0,20 \mathrm{~B}$ & $10,49 \pm 0,26 \mathrm{~B}$ \\
\hline
\end{tabular}

Médias seguidas de mesma letra na vertical não diferem $(\mathrm{P}<0,05)$ entre si pelo teste Tukey.

$\mathrm{ART}=$ alimento vivo por todo período experimental; JJ = jejum; DI = dieta comercial importada; DPN $=$ dieta comercial nacional; DMC = dieta experimental microencapsulada; ADI, ADPN e ADMC = náuplios de artêmia durante os seis primeiros dias experimentais, seis dias de coalimentação, e somente as respectivas dietas formuladas após esse período. 


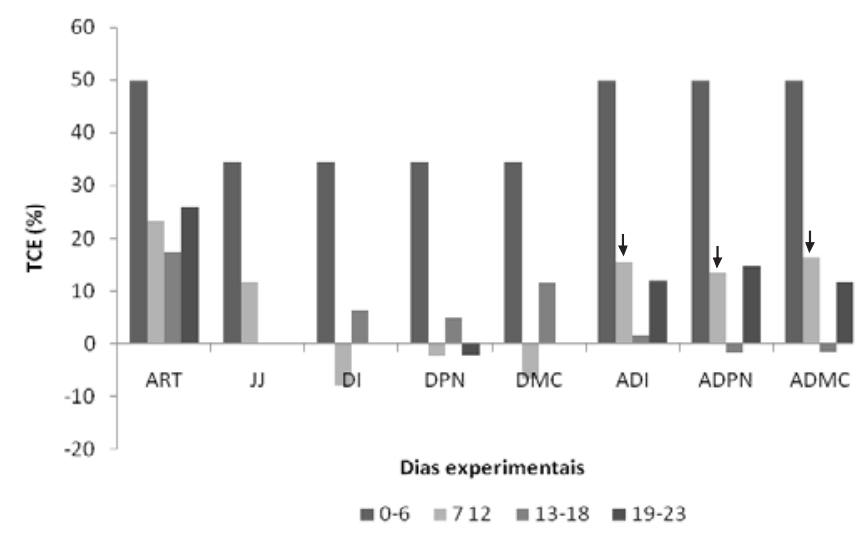

$\mathrm{ART}=$ náuplios de artêmia por todo período experimental; $\mathrm{JJ}=$ jejum; DI = dieta comercial importada; DPN = dieta comercial nacional; DMC $=$ dieta experimental microencapsulada; ADI, ADPN e ADMC = náuplios de artêmia durante os seis primeiros dias experimentais, seis dias de coalimentação e somente as respectivas dietas formuladas após esse período. As setas indicam o início da transição alimentar.

Figura 3 - Valores médios (\%) da taxa de crescimento específico das larvas de pacu submetidas aos diferentes protocolos alimentares.

comprimento de larvas de pacu após a substituição do alimento vivo pelo inerte, independentemente da idade do início da transição e retomada do crescimento após alguns dias.

Além disso, durante o período de alimentação mista ( $7 \underline{\mathrm{o}}$ a $12^{\circ}$ dia), quando a oferta de náuplios foi diminuída gradualmente, houve decréscimo da taxa de crescimento específico dos animais que tiveram o alimento vivo substituído pelas dietas inertes (Figura 3). Mesmo com aumento de peso muito acentuado (83,5 vezes em 23 dias), as larvas do controle positivo também apresentaram queda da taxa de crescimento específico, uma tendência esperada já que, em geral, no início do desenvolvimento, o crescimento ocorre em ritmo mais acelerado e, à medida que os organismos crescem, as taxas de crescimento diário diminuem. Portella (2000) verificou redução da taxa de crescimento de juvenis de Prochilodus scrofa no período de 31 a 35 dias de vida em relação aos valores apresentados até os 30 primeiros dias, mesmo com manutenção da oferta de alimento vivo. Resultados similares, indicando diminuição da taxa de crescimento dos peixes, foram relatados por Dabrowski \& Poczyczynski (1988) em larvas de Coregnus larvaretus. Esses autores observaram que, nos dez primeiros dias de vida, a taxa de crescimento específico era de $18,4 \%$, nos dez dias subsequentes de $9,9 \%$ e entre o 19 e e 43 o dia de $6,4 \%$.

$\mathrm{Na}$ avaliação seguinte (18 dias), os valores médios de comprimento e peso das larvas alimentadas com alimento vivo foram significativamente superiores $(\mathrm{P}<0,05)$ aos das larvas submetidas à transição alimentar, ou seja, que receberam alimento vivo somente nos primeiros 12 dias de experimento ou os que receberam apenas as dietas formuladas (Tabelas 1 e 2). Na última biometria (23 dias), ficou evidente que as larvas de pacu nessa fase de desenvolvimento ainda necessitam de suprimento de alimento vivo. Os maiores valores de peso e comprimento foram encontrados nas larvas alimentadas com náuplios de artêmia, seguido de resultados intermediários das larvas que tiveram esse alimento suprimido durante a fase de transição alimentar, independentemente do tipo da dieta formulada fornecida (Tabelas 1 e 2). As larvas que receberam exclusivamente a ração comercial nacional praticamente não aumentaram em peso e em comprimento durante todo o período experimental. Segundo Garcia-Ortega et al. (2003), maior crescimento é alcançado quando as larvas recebem alimento vivo por um período mais longo.

Foi verificado visualmente e também em cortes histológicos (Figuras 4A e 5F) que as larvas tratadas exclusivamente com as dietas formuladas ingeriram o alimento. Todavia, o baixo aproveitamento de dietas inertes como primeira fonte de alimentação de larvas de peixes pode estar diretamente ligado à capacidade digestiva das larvas de peixes, que, em geral, apresentam o aparelho digestório ainda indiferenciado no início da alimentação exógena (Hachero-Cruzado et al., 2009) e ausência de algumas enzimas digestivas. Diversos autores argumentam que essas características são os principais fatores limitantes para a utilização de microdietas nos primeiros estágios do desenvolvimento larval (Dabrowski, 1984; Moyano et al., 1996). Por outro lado, dietas inertes são comumente compostas por carboidratos complexos e proteínas desnaturadas, insolúveis ou pouco solúveis, que formam uma partícula densa e com alto peso molecular (Langdon, 2003). Isso torna esses alimentos química e fisicamente muito diferentes do alimento vivo, que possui os nutrientes dispersos em matriz aquosa. Conceição et al. (2010) sustentam que, independentemente do seu valor nutricional, o alimento vivo é mais facilmente detectado e capturado pelas larvas, devido à sua natação na coluna d'água, e apresentam maior digestibilidade e palatabilidade quando comparados às dietas formuladas, que, geralmente, são mais duras e contêm maior quantidade de matéria seca. Além disso, os organismos vivos apresentam perfil de aminoácidos mais adequado e são de mais fácil digestão para as larvas de peixes (Langdon, 2003), além de conter outras substâncias, como neuropeptídios e fatores de crescimento, que propiciam o desenvolvimento do trato digestório (Kolkovski, 2001). Pela combinação de todas essas características, aliada ao desconhecimento dos fatores presentes no alimento vivo que favorecem o pleno desenvolvimento larval, torna difícil sua substituição completa nos sistemas intensivos de criação de larvas de peixes. 
No início do experimento, as larvas ainda apresentavam um pouco de reserva vitelina. O tubo digestivo era retilíneo e o epitélio simples, ainda indiferenciado, formado por células com núcleos basais. No fígado não havia evidências de acúmulo de glicogênio. Aos seis dias de experimento, as larvas que receberam somente as dietas inertes como primeira fonte de alimento (independentemente da dieta) apresentavam algumas pregas longitudinais na região do esôfago, separando a região do futuro estômago, ainda indiferenciada, da porção anterior do intestino, mais dilatada (Figura 4A). Aos 12 dias experimentais, pela reação ao ácido periódico de Schiff, não foi observada presença de reservas de glicogênio no fígado de larvas em jejum (Figura 6A) nem das que receberam somente dietas formuladas (Figura 6B), nas quais, no entanto, detectaram-se vacuolizações evidentes, possivelmente sinais de má nutrição. Na parte mais distal do tudo digestivo dessas larvas, não foram observadas inclusões supranucleares nos enterócitos (Figura 5B) e o epitélio intestinal assemelhava-se ao das larvas mantidas em jejum (Figura 5A).

Já nos animais que receberam alimento vivo nos primeiros seis dias experimentais, o trato digestivo encontrava-se em estágio mais avançado de maturação. Aos seis dias, a região do futuro estômago apresentava maior quantidade de pregas (Figura 4B) e, aos 12 dias, no intestino as vilosidades eram mais evidentes e os enterócitos exibiam maior vacuolização (Figura $5 \mathrm{C}$ ). O fígado já apresentava acúmulo de glicogênio, ácido periódico de Schiff(Figura 6C) e, no pâncreas, foram observados ácinos bem definidos com grânulos de zimogênio, corados pelo método eosina-orange G-floxina. Na observação realizada no final do período de transição alimentar (12으 dia), foi possível observar que as larvas que tiveram os náuplios substituídos pelas dietas inertes apresentaram-se em uma fase um pouco anterior em relação à observada nas larvas do controle positivo, que recebia náuplios de artêmia.

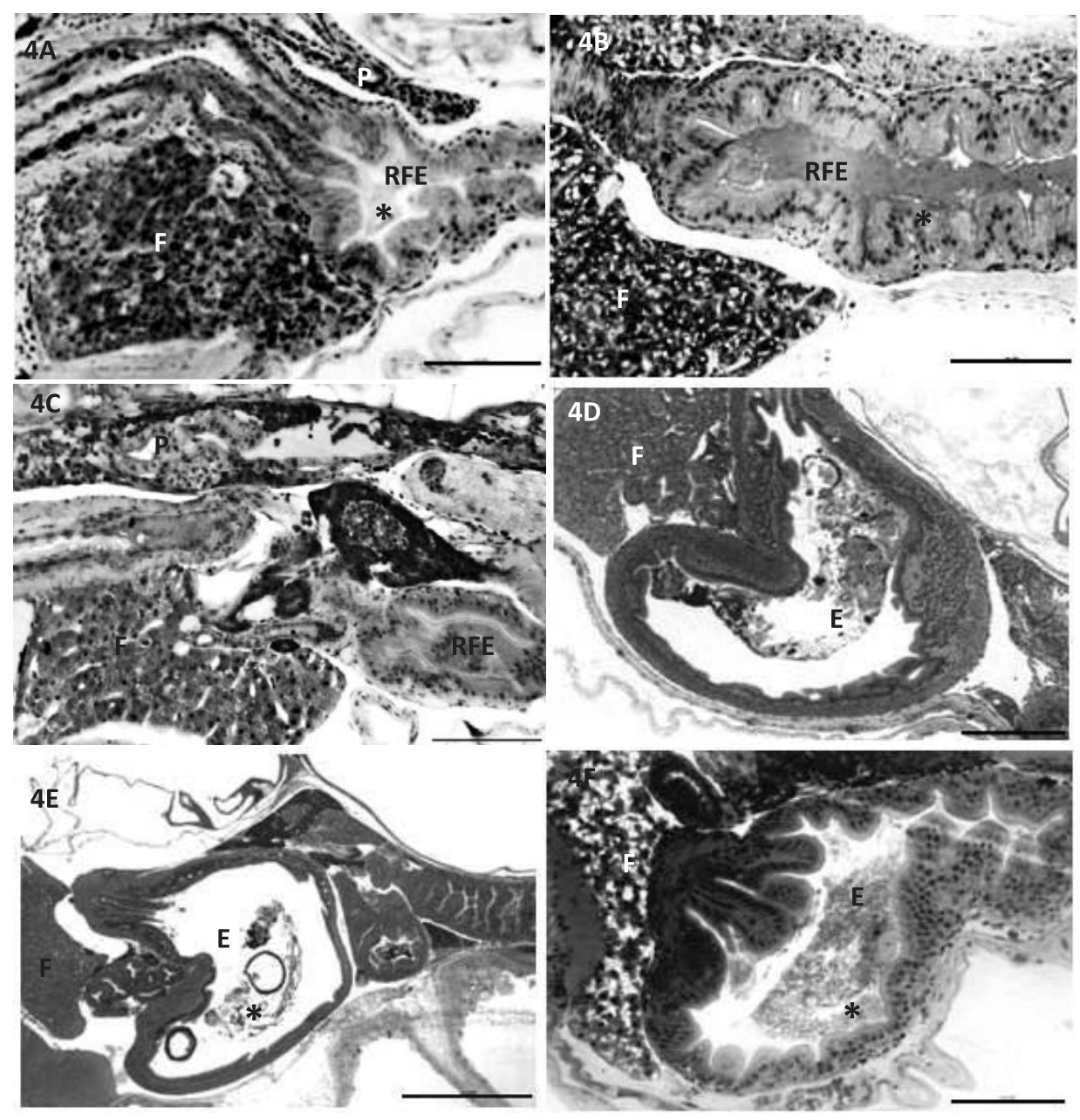

4A: larva no 6o dia alimentada com dieta microencapsulada; HE. Barra $100 \mu \mathrm{m}$; 4B: larva no 6o dia alimentada com náuplios de artêmia; HE. Barra 100 $\mu$ m; 4C: larva em jejum no $6 \underline{0}$ dia; HE. Barra $100 \mu \mathrm{m}$; 4D: larva no 23ㅇ dia alimentada com náuplios de artêmia; AM. Barra 200 $\mu \mathrm{m}$; 4E: larva no 23o dia que passou pela transição alimentar com a dieta microencapsulada; AM. Barra $100 \mu \mathrm{m} ; 4 \mathrm{~F}$ : larva no 230 dia alimentada com a dieta nacional; AM. F = fígado; RFE = região presuntiva do futuro estômago; $\mathrm{E}=$ estômago; $\mathrm{P}=$ pâncreas; $*$ conteúdo alimentar.

Figura 4 - Região do futuro estômago (4A, 4B e 4C) e estômago (4D, 4E e 4F) de larvas de pacu em diferentes dias experimentais. 
Nessas últimas, o intestino apresentou padrão similar ao da última coleta; porém, por meio de análise comparativa e subjetiva, constatou-se que apresentavam vilosidades mais altas e com maior quantidade de inclusões supranucleares. No pâncreas observou-se grande quantidade de grânulos de zimogênio, indicando intensa atividade secretora das células que formam as glândulas exócrinas do pâncreas. Nas larvas em que eram fornecidas apenas as dietas formuladas, não houve diferenças histológicas notáveis em comparação à coleta anterior, aos seis dias, indicando que praticamente não houve desenvolvimento morfológico das estruturas digestórias e que a substituição alimentar não favoreceu o pleno desenvolvimento do trato digestório das larvas de pacu nessa fase da vida.

Baixa ingestão, digestão e assimilação das microdietas são razões frequentemente apontadas para explicar o desempenho insatisfatório de larvas que não recebem alimento vivo (Curnow et al., 2006). Papadakis et al. (2009) sugeriram que a taxa de maturação dos vários órgãos que formam o sistema digestório pode ser influenciada pelos diferentes tipos de alimentos. Esses autores afirmaram que esse fato deve ser considerado para a formulação de dietas e para a implantação de novos protocolos alimentares para larvas de peixes. A quantidade de matéria seca presente nas partículas é um fator que contribui para dificultar sua assimilação pelas larvas. Diferentemente do alimento vivo, que possui cerca de $10 \%$ de matéria seca, dietas inertes possuem por volta de $60 \%$ (Kolkovski, 2001), tornando essas dietas mais difíceis de ser hidrolisadas e digeridas.

Ao final do experimento, as larvas alimentadas somente com artêmia destacaram-se, não somente por seu melhor desempenho zootécnico, mas também pela organogênese de seu sistema digestório, que estava notavelmente mais desenvolvido que o das demais larvas. Somente na coleta

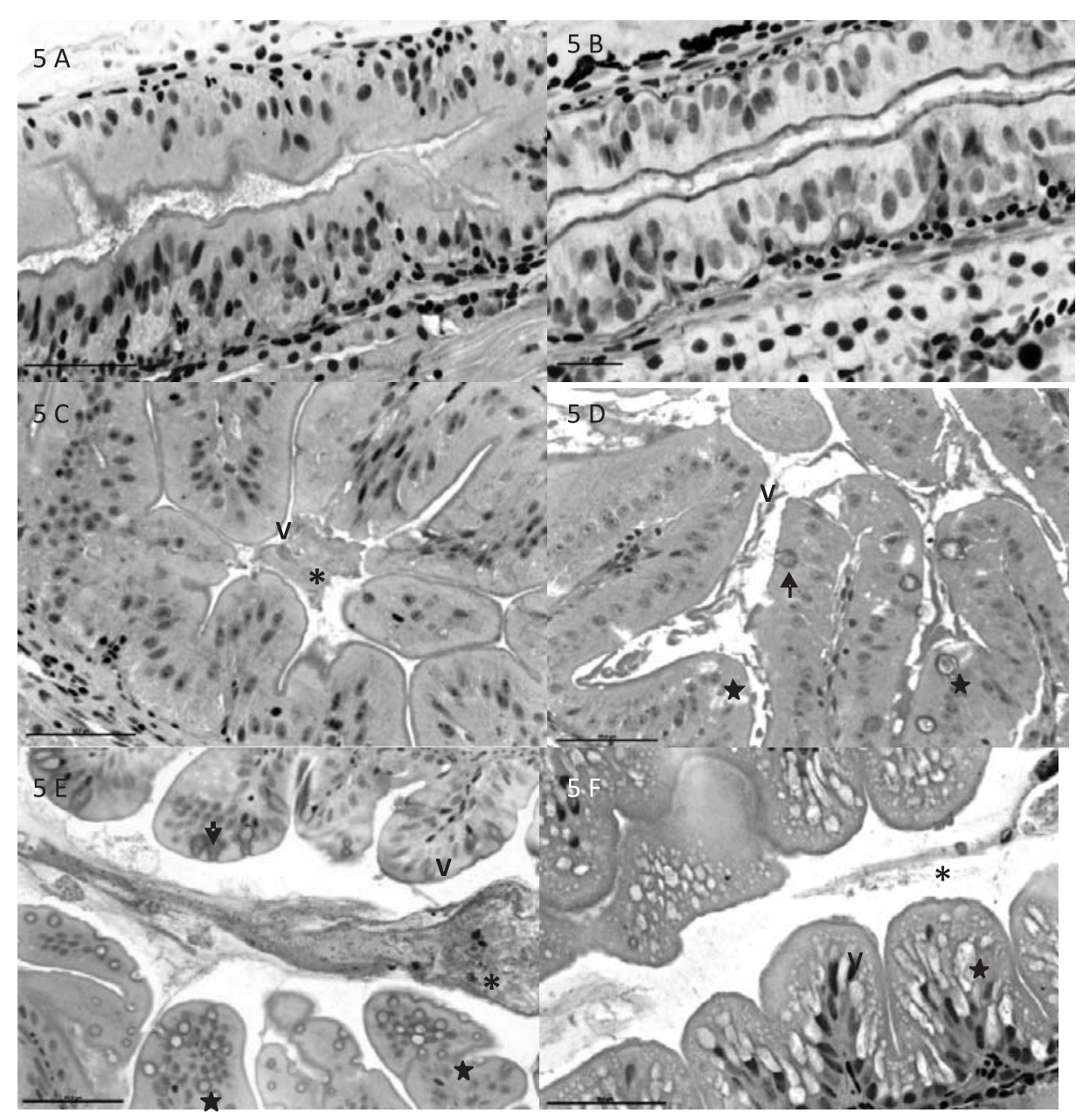

5A: larva no $12^{\mathrm{o}}$ dia mantida em jejum; HE. Barra $50 \mu \mathrm{m} ; 5 \mathrm{~B}$ : larva no $12^{\circ} \mathrm{o}$ dia alimentada com dieta microencapsulada; PAS. Barra $20 \mu \mathrm{m}$; $5 \mathrm{C}$ : 1arva no $12^{\circ}$ dia que passou

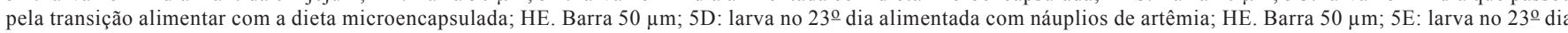
que passou pela transição alimentar com a dieta microencapsulada; HE. Barra $50 \mu \mathrm{m}$; 5F: larva no 23 o dia alimentada com dieta inerte (DPN); HE. Barra $50 \mu \mathrm{m}$. Vilosidades (V), células caliciformes (seta) e inclusões supranucleares $(\star)$, conteúdo alimentar ${ }^{*}$ ).

Figura 5 - Intestino de larvas de pacu em diferentes dias experimentais. 
realizada aos 23 dias, foram observadas glândulas gástricas, que eram mais abundantes no controle positivo (Figura 4D) que naqueles que passaram pela transição alimentar (Figura 4E) ou com uso exclusivo da dieta nacional (Figura 4F). Tanto nas larvas que receberam apenas o alimento vivo (Figura 5D) quanto naquelas que tiveram esse alimento substituído (Figura 5E), o epitélio do intestino apresentava vilosidades e inclusões supranucleares, com abundância de células caliciformes, em maior quantidade nas que ingeriram apenas náuplios de artêmia. No fígado dessas larvas, estavam presentes reservas de glicogênio (Figuras 6D e 6E). Assim como observado neste estudo, já foram relatados casos de atraso ontogênico do sistema digestório de larvas de diferentes espécies quando a transição alimentar foi realizada precocemente (Person Le Ruyet, 1989; Hart \& Purser, 1996; Jomori, 2005; Tesser et al., 2006; Engrola et al., 2009).
Entre as larvas que receberam somente dietas inertes, o único grupo sobrevivente ao final do experimento foi aquele alimentado com a dieta nacional. No final do experimento ( 23 dias), houve pequeno progresso no desenvolvimento do sistema digestório dessas larvas (Figura 4F), já que no intestino aparentemente havia mais vilosidades e indicações de sinais de absorção da dieta (Figura 5F) em relação às coletas prévias. Observou-se reserva de glicogênio (ácido periódico de Schiff + ) no fígado dessas larvas, evidência que não foi observada nas coletas anteriores (Figura 6F). Segundo Cahu \& Zambonino-Infante (2001), algumas enzimas digestivas já estão presentes no início da alimentação exógena. A atividade dessas enzimas é geneticamente programada, mas o fornecimento de alimentos que não satisfaçam às necessidades nutricionais das larvas pode retardar ou mesmo impedir a programação genética da maturação intestinal (Cahu \& Zambonino-Infante, 2001).

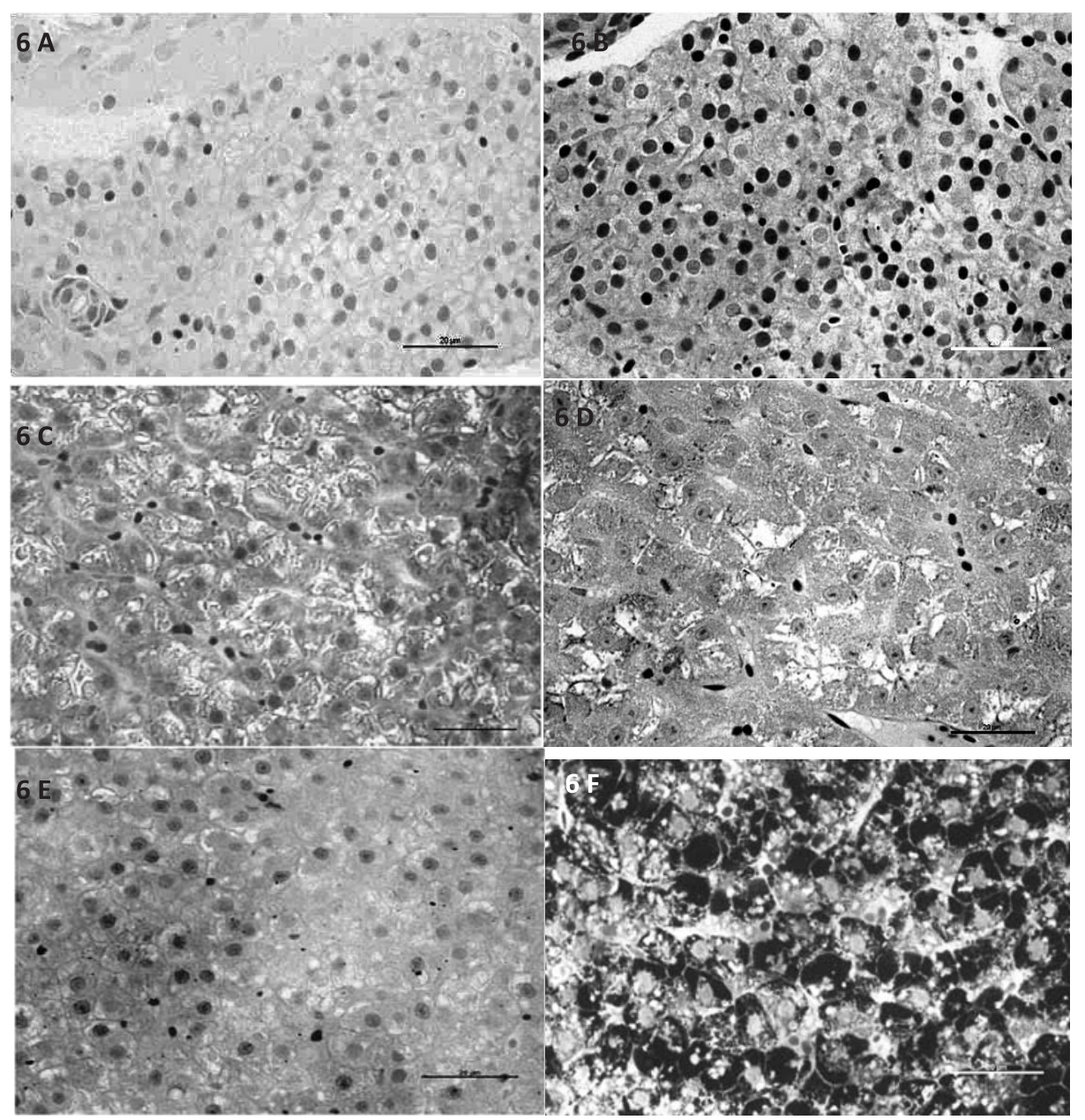

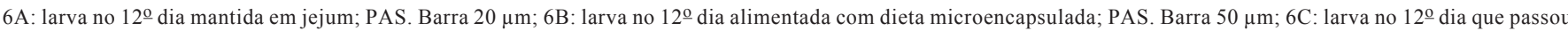
pela transição alimentar com a dieta experimental microencapsulada; PAS. Barra $50 \mu \mathrm{m}$; $6 \mathrm{D}$ : larva no 23 o dia alimentada com náuplios de artêmia; PAS. Barra $50 \mu \mathrm{m}$; 6E: larva no 23으 dia que passou pela transição alimentar com a dieta experimental microencapsulada (ADMC); PAS. 6F: larva no 23ㅇ dia alimentada com dieta inerte (DPN); PAS. Barra $50 \mu \mathrm{m}$

Figura 6 - Fígado de larvas de pacu em diferentes dias experimentais. 
Nenhuma das microdietas testadas foi capaz de sustentar o crescimento nas fases iniciais, corroborando os resultados observados em outros trabalhos com larvas de pacu (Jomori, 2005; Tesser et al., 2005; Jomori et al., 2008; Leitão et al., 2011) e outros com espécies marinhas (Fernández-Díaz \& Yúfera, 1997; Yúfera et al., 2005), indicando que não são adequadas para uso como único alimento nas fases iniciais de desenvolvimento de pacu. Entretanto, a obtenção de larvas vivas ao final do experimento indica que mais investimentos científicos e tecnológicos para a melhoria da formulação e tecnologia de produção das microdietas abrem perspectivas para reduzir, com êxito, o período de fornecimento do alimento vivo, visando produção mais econômica de larvas de peixes.

\section{Conclusões}

A eficiência da dieta inerte como alimento inicial para larvas de pacu ainda está fortemente relacionada à presença do alimento vivo. As dietas (experimental e comerciais) avaliadas nesta pesquisa não são adequadas para fornecimento como alimento exclusivo para larvas de pacu. O não-fornecimento ou a supressão muito precoce da oferta de náuplios de artêmia prejudica o crescimento e o desenvolvimento do sistema digestório de larvas, no entanto, a presença de larvas vivas ao final do experimento confirma a necessidade de mais pesquisas para melhorar a formulação e a tecnologia de produção de microdietas visando à redução do período de fornecimento de alimento vivo para maior produtividade e economia na larvicultura de espécies neotropicais em sistemas intensivos de criação.

\section{Agradecimentos}

Ao CNPq, pela bolsa de mestrado concedida ao primeiro autor.

\section{Referências}

CAHU, C.L.; ZAMBONINO INFANTE, J.L. Substitution of live food by formulated diets in marine fish larvae. Aquaculture, v.200, p.161-80, 2001

CONCEIÇÃO, L.E.C.; YÚFERA, M.; MAKRIDIS, P. et al. Live feed for early stages of fish rearing. Aquaculture Research, v.41, p.613-640, 2010

CURNOW, J.; KING, J.; PARTRIDGE, G. et al. Effects of two commercial microdiets on growth and survival of barramundi (Lates calcarifer Bloch) larvae within various early weaning protocols. Aquaculture Nutrition, v.12, p.247-255, 2006.

DABROWSKI, K. The feeding of fish larvae: present "state of art" and perspectives. Reproduction Nutrition Development, v. 24, n.6, p.807-833, 1984.
DABROWSKI, K.; POCZYCZYÑSKI, P.J. Laboratory experiment and mass rearing of coreogonid fish fed exclusively on dry diets. Aquaculture, v.69, p.307-316, 1988.

ENGROLA, S.; FIGUEIRA, L.; CONCEIÇÃO, E.C.L. et al. Co-feeding in Senegalese sole larvae with inert diet from mouth opening promotes growth at weaning. Aquaculture, v.288, p.264-272, 2009.

FERNÁNDEZ-DÍAZ, C.; YÚFERA, M. Detecting growth in gilthead seabream, Sparus aurata L., larvae fed microcapsules. Aquaculture, v.153, p.93-102, 1997.

GARCÍA-ORTEGA, A.; ABDO, I; HERNÁNDEZ, C. Weaning of bullseye puffer (Sphoeroides annulatus) from live food to microparticulate diets made with decapsulated cysts of Artemia and fishmeal. Aquaculture International, v.11, p.183-194, 2003.

HACHERO-CRUZADO, I.; ORTIZ-DELGADO, J.B.; BORREGA, B et al. Larval organogenesis of flatfish brill Scophthalmus rhombus L: Histological and histochemical aspects. Aquaculture, v.286, p.138-149, 2009.

HART, P.R.; PURSER, G.J. Weaning of hatchery-reared greenback flounder (Rhombosolea tapirina Günther) from live to artificial diets: Effects of age and duration of the changeover period. Aquaculture, v.145, p.171-181, 1996.

JOMORI, R.K. Organismos vivos e dietas secas na larvicultura do pacu Piaractus mesopotamicus e uso dos isótopos estáveis de carbono e nitrogênio como indicadores naturais da incorporação do alimento no tecido larval. 2005. $121 \mathrm{f}$ Tese (Doutorado em Aqüicultura de Águas Continentais) UNESP, Jaboticabal

JOMORI, R.K.; CARNEIRO, D.J.; MALHEIROS, E.B. et al. Growth and survival of pacu Piaractus mesopotamicus (Holmberg, 1887) juveniles reared in ponds or at different initial larviculture periods indoors. Aquaculture, v.221, p.277-287, 2003.

JOMORI, R.K.; DUCATTI, C.; CARNEIRO, D.J. et al. Stable carbon $(\mathrm{d} 13 \mathrm{C})$ and nitrogen $(\mathrm{d} 15 \mathrm{~N})$ isotopes as natural indicators of live and dry food in Piaractus mesopotamicus (Holmberg, 1887) larval tissue. Aquaculture Research, v.237, p.1-12, 2008.

KESTEMONT, P.; STALMANS, J.M. Initial feeding of European minnow larvae Phoxinus phoxinus L. Influence of diet and feeding level. Aquaculture, v.104, p.327-340, 1992.

KOLKOVSKI, K. Digestive enzymes in fish larvae and juveniles implication and applications to formulated diet. Aquaculture, v.200, p.181-201, 2001

KOVEN, W.; KOLKOVSKI, W.; HADAS. E. et al. Advances in the development of microdiets for gilthead seabream, Sparus aurata: a review. Aquaculture, v.194, p.107-121, 2001.

LANGDON, C.J. Microparticle types for delivering nutrients to marine fish larvae. Aquaculture, v.227, p.259-275, 2003.

LEITÃO, N.J.; PAI-SILVA, M.D.; ALMEIDA, F.L.A. et al. The influence of initial feeding on muscle development and growth in pacu Piaractus mesopotamicus larvae. Aquaculture, v.315, p.78-85, 2011

MOYANO, F.J.; DIAZ, M.; ALARCÓN, F.J. et al. Characterization of digestive enzyme activity during larval development of guilthead seabrem Sparus aurata. Fish Physiology Biochemistry, v.15, p.121-130, 1996

PAPADAKIS, I.E.; ZAISS, M.M.; KYRIAKOU, Y. et al. Histological evaluation of the elimination of Artemia nauplii from larval rearing protocols on the digestive system ontogeny of shi drum (Umbrina cirrosa L.) Aquaculture, v.286, p.45-52, 2009.

PERSON LE-RUYET, J. Early weaning of marine fish larvae onto microdiets: constraints and perspectives. Advances in tropical aquaculture. Tahiti. Fev 20 - March 4. 1989. AQUACOP INFREMER. Actes de Colloque, n.9, p.625-642, 1989.

PORTELLA, M.C.; VERANI, J.R.; CESTAROLLI, M.A. Use of live and artificial diets enriched with several fatty acids sources to feed Prochilodus scrofa larvae and fingerlings. 1. Effects on survival and growth. Journal of Aquaculture in the Tropics, v. 15, n. 1, p. $45-58,2000$. 
PORTELLA, M.C.; DABROWSKI, K. Diets, physiology, biochemistry and digestive tract development of freshwater fish larvae. In: CYRINO, J.E.C.; BUREAU, D.; KAPOOR, B.G. (Orgs.). Feeding and digestive functions of fishes. Enfield: Science Publishers, 2008. p.227-279.

TESSER, M.B.; CARNEIRO, D.J.; PORTELLA, M.C. Co-feeding of pacu, Piaractus mesopotamicus Holmberg (1887), larvae with Artemia nauplii and a microencapsulated diet. Journal of Applied Aquaculture, v.17, n.2, p.47-59, 2005.

TESSER, M.B.; FLORES-QUINTANA, C.I.; CARNEIRO, D.J. et al. Suplementação de enzimas exógenas em dieta microparticulada para larvicultura do pacu. Revista Brasileira de Zootecnia, v.35, p.2211-2218, 2006.

YÚFERA, M.; PASCUAL, E.; FERNÁNDEZ-DIAZ, C. A highly efficient microencapsulated food for rearing early larvae of marine fish. Aquaculture, v.177, p.249-256, 1999.

YÚFERA, M.; FERNÁNDEZ-DÍAS, C.; PASCUAL, E. Food microparticules for larval fish prepared by internal gelation. Aquaculture, v.248, p.253-262, 2005.

YÚFERA, M.; PORTELLA, M.C.; DRUST, S. et al. Microencapsulation and Aquaculture. Aquaculture Europe, v.34, p.18-20, 2009. 